\title{
Spin tunneling and topological selection rules for integer spins
}

\author{
Michael N. Leuenbergent and Daniel Loss \\ Department of Physics and Astronomy, University of Basel \\ Klingelbergstrasse 82, 4056 Basel, Switzerland
}

\begin{abstract}
We present topological interference effects for the tunneling of a single large spin, which are caused by the symmetry of a general class of magnetic anisotropies. The interference originates from spin Berry phases associated with different tunneling paths exposed to the same dynamics. Introducing a generalized path integral for coherent spin states, we evaluate transition amplitudes between ground as well as low-lying excited states. We show that these interference effects lead to topological selection rules and spin-parity effects for integer spins that agree with quantum selection rules and which thus provide a generalization of the Kramers degeneracy to integer spins. Our results apply to the molecular magnets $\mathrm{Mn}_{12}$ and $\mathrm{Fe}_{8}$.
\end{abstract}

PACS numbers: 75.45.+j, 03.65.Vf, 75.10.Dg

In recent years the tunneling of spin has attracted much attention since experiments provided strong evidence for the existence of spin tunneling in molecular magnets, such as $\mathrm{Mn}_{12}$-acetate $\left(\mathrm{Mn}_{12}\right)$ 道远

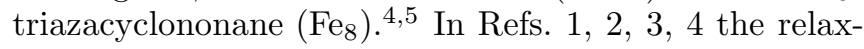
ation time $\tau\left(H_{z}\right)$ of the magnetization as a function of the longitudinal magnetic field $H_{z}$ was measured, with $\tau\left(H_{z}\right)$ exhibiting large peaks due to thermal-assisted tun neling of the spin.6 In Ref. 5 , the incoherent Zener rate as a function of the transverse magnetic field $H_{x}$ was measured, from which quantum oscillations of the tunnel splitting $E_{m m^{\prime}}\left(H_{x}\right)$ can be observed. $\mathrm{In}$ a coherent spin-state path-integral approach, 8 these quantum oscillations and their associated spin-parity effects in the case of quadratic anisotropies can be viewed as a result of interfering Berry phasescarried by spin tunneling paths of opposite windings, 1011 which are modified in the presence of a transverse-field $H_{x}$ (Ref. 12) or crystal fields of cubic symmetry with multiple degenerate minima. 13

The results obtained in the present work can be summarized as follows. If the Hamiltonian for a single spin $s \gg 1$ contains a magnetic anisotropy of arbitrary order $n$ that is transverse to the easy axis and symmetric under rotations of $2 \pi / n$ around the easy axis, there are $n$ different paths that possess the same dynamical action but different spin Berry phases and which thus interfere constructively or destructively. Using a spin path integral that we generalize to excited states we will show that the tunneling of the spin between ground or low-lying excited states that are doubly degenerate, $|m\rangle$ and $|-m\rangle$, $|m| \leq s$, is completely suppressed if $n$ is not a divisor of $2 m$ (i.e., $2 m / n \notin \mathbb{Z}$ ); this result represents a generalization of the Kramers degeneracy to integer spins $s$. We also provide the connection to quantum selection rules using standard spin algebra, and we discuss the effect of magnetic fields on these selection rules.

We consider a general single-spin Hamiltonian $\mathcal{H}_{z, n}=$ $-A S_{z}^{2}+B_{n}\left(S_{+}^{n}+S_{-}^{n}\right)$, with easy-axis $(A)$ and transverse $\left(B_{n}\right)$ anisotropy constants satisfying $A \gg B_{n}>$ 0 . Here, $n$ is an even integer, i.e., $n=2,4,6, \ldots$, so that $\mathcal{H}_{z}$ is invariant under time reversal. Such Hamiltonians are relevant for molecular magnets such as $\mathrm{Mn}_{12}$ (Refs. 6 and ) and $\mathrm{Fe}_{8}$ 目8 The corresponding classical anisotropy energy $E_{z, n}(\theta, \phi)=-A s^{2} \cos ^{2} \theta+$ $B_{n} s^{n} \sin ^{n} \theta \cos (n \phi)$ has the shape of a double-well potential, with the easy axis pointing along the $z$ direction. It is obvious that the anisotropy energy remains invariant under rotations around the $z$ axis by multiples of the angle $\eta=2 \pi / n$, i.e., $\mathcal{R}_{z, \eta} E_{z}(\theta, \phi)=$ $E_{z}(\theta, \phi+\eta)=E_{z}(\theta, \phi)$. It is convenient to describe the symmetry of the anisotropy energy by the cyclic group $C_{\alpha, n}=\left\{\mathcal{R}_{\alpha, \eta}, \mathcal{R}_{\alpha, 2 \eta}, \ldots, \mathcal{R}_{\alpha,(n-1) \eta}, \mathcal{R}_{\alpha, n \eta}=\mathcal{I}\right\}$, where $\mathcal{R}_{\alpha, \varphi}$ performs rotations about the $\alpha$-axis, $\alpha=x, y, z$, by the angle $\varphi$, and $\mathcal{I}$ denotes the identity operator.

For the following calculation it proves favorable to choose the easy axis along the $y$ direction. Then the spin Hamiltonian and the anisotropy energy are changed into

$$
\mathcal{H}_{y, n}=-A S_{y}^{2}+B_{n}\left(S_{+}^{n}+S_{-}^{n}\right),
$$

where now $S_{ \pm}=S_{z} \pm i S_{x}$, and

$$
\begin{aligned}
E_{y, n}(\theta, \phi)= & -A s^{2} \sin ^{2} \theta \sin ^{2} \phi+B_{n} s^{n}[(\cos \theta \\
& \left.+i \sin \theta \cos \phi)^{n}+(\cos \theta-i \sin \theta \cos \phi)^{n}\right] .
\end{aligned}
$$

We note again that $C_{y, n} E_{y, n}=E_{y, n}$, which will play a central role in what follows.

We are interested in the tunneling between the eigenstates $|m\rangle$ and $|-m\rangle$ of $-A S_{y}^{2}$, corresponding to the global minimum points $(\theta=\pi / 2, \phi=-\pi / 2)$ and $(\theta=$ $\pi / 2, \phi=+\pi / 2)$ of $E_{y, n}$. For this we evaluate the imaginary time transition amplitude between these points. For the ground-state tunneling $(m=s \gg 1)$ this can bedone by means of the coherent spin-state path integral 10

$$
\left\langle-\frac{\pi}{2}\left|e^{-\beta \mathcal{H}}\right|+\frac{\pi}{2}\right\rangle=\int_{\pi / 2,-\pi / 2}^{\pi / 2,+\pi / 2} \mathcal{D} \Omega e^{-S_{\mathrm{E}}}
$$

where $\beta=1 / k_{B} T$ is the inverse temperature, $\mathcal{D} \Omega=$ $\Pi_{\tau} d \Omega_{\tau}, d \Omega_{\tau}=[4 \pi /(2 s+1)] d\left(\cos \theta_{\tau}\right) d \phi_{\tau}$ the Haar measure of the $S^{2}$ sphere, and $S_{\mathrm{E}}=\int_{0}^{\beta} d \tau[i s \dot{\phi}(1-\cos \theta)+$ $\left.E_{y, n}\right]$ the Euclidean action, where the first term in $S_{\mathrm{E}}$ defines the Wess-Zumino (or Berry phase) term, 14 which gives rise to topological interference effects for spin tunneling. 10112123 


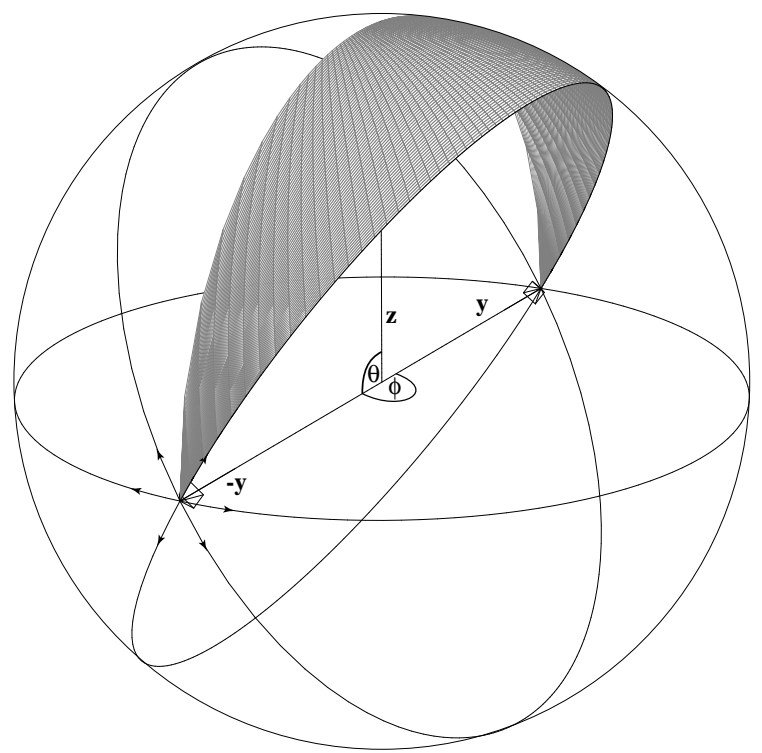

FIG. 1. Interference between $n=6$ paths (small arrows) moving from $\mathbf{- y}$ to $\mathbf{y}$ direction (large arrows), which is destructive if $n$ is not a divisor of $2 m$. The grey surface area $\Phi(m) / m$ depicts the Berry phase $\Phi(m)$ of the upper right path. Note that there is no simple pairwise interference; instead, all six paths interfere with each other [see Eq. (\$)].

For the tunneling between low-lying excited states $(1 \ll|m| \lesssim s)$ we now derive a generalized coherent spin-state path integral. It is known 15 that one can construct a coherent state system $\left\{T\left|\psi_{0}\right\rangle\right\}$ out of any starting vector $\left|\psi_{0}\right\rangle$ of a Hilbert space $\mathbb{H}$, with $T$ being a representation of any Lie group operating on this $\mathbb{H}$. Thus, applying this to our case, we can choose any spin state $|m\rangle \in \mathbb{H}_{s}=\{|m\rangle \mid-s \leq m \leq s\}$ as starting vector together with the Lie group $S U_{2}$, leading to the generalized coherent spin states

$$
|\boldsymbol{\Omega}, m\rangle=e^{-i S_{z} \phi} e^{-i S_{y} \theta} e^{-i S_{z} \chi}|m\rangle,
$$

where $\phi, \theta$, and $\chi$ are the Euler angles. Note that the dispersion of $\mathbf{S}^{2}$ is not minimal for $|m\rangle \neq|s\rangle$. However, as long as $1 \ll|m| \lesssim s$, the spin fluctuations remain small. By means of Schur's Lemma, one can prove as in Ref. 15 that the integral $\int d \Omega|\boldsymbol{\Omega}, m\rangle\langle\boldsymbol{\Omega}, m| \propto$ $\mathcal{I}$. So we have to calculate one single diagonal element in order to fix the proportionality constant. For this it turns out to be convenient to express the states $|\boldsymbol{\Omega}, m\rangle$ by $2 s$ spin- $1 / 2$ states that are individually rotated, i.e., $\left|\uparrow^{\prime}\right\rangle=\cos (\theta / 2)|\uparrow\rangle+e^{i \phi} \sin (\theta / 2)|\downarrow\rangle,\left|\downarrow^{\prime}\right\rangle=$ $e^{-i \phi} \sin (\theta / 2)|\uparrow\rangle-\cos (\theta / 2)|\downarrow\rangle$, where the singee-valued states are represented in the north pole gauge. 16 Thus, we obtain

$$
|\boldsymbol{\Omega}, m\rangle=\left(\begin{array}{c}
2 s \\
s-m
\end{array}\right)^{-1 / 2} \sum_{\substack{j_{1}<\cdots<j_{s-m} \\
1 \leq j_{k} \leq 2 s}}\left|\uparrow_{1}^{\prime} \cdots \downarrow_{\left\{j_{k}\right\}}^{\prime} \cdots \uparrow_{2 s}^{\prime}\right\rangle,
$$

where $\downarrow_{\left\{j_{k}\right\}}^{\prime}$ denotes the $s-m$ rotated down spins $\left|\downarrow_{j_{k}}^{\prime}\right\rangle$, at the positions $j_{k}$. Evaluation of the amplitude

$$
\begin{aligned}
\langle\boldsymbol{\Omega}, m \mid s\rangle & =\left\langle\boldsymbol{\Omega}, m \mid \uparrow_{1} \uparrow_{2} \cdots \uparrow_{2 s}\right\rangle \\
& =\sqrt{\left(\begin{array}{c}
2 s \\
s-m
\end{array}\right)}\left(\cos \frac{\theta}{2}\right)^{s+m}\left(e^{i \phi} \sin \frac{\theta}{2}\right)^{s-m}
\end{aligned}
$$

leads to $\int d \Omega\langle s \mid \boldsymbol{\Omega}, m\rangle\langle\boldsymbol{\Omega}, m \mid s\rangle=4 \pi /(2 s+1)$. Hence, the integration measure for all $|\boldsymbol{\Omega}, m\rangle$ states is the same. Next, to derive the path integral, we need the quantity $\langle\tilde{\boldsymbol{\Omega}}, m|\mathbf{S}| \boldsymbol{\Omega}, m\rangle=(m \boldsymbol{\Omega}+\mathcal{O}(\sqrt{s}))\langle\tilde{\boldsymbol{\Omega}}, m \mid \boldsymbol{\Omega}, m\rangle, 17$ where we must choose the value of $m$ close to $s$. Consequently, we have to evaluate the overlap between two states separated by the angles $\delta \theta=\tilde{\theta}-\theta$ and $\delta \phi=\tilde{\phi}-\phi$,

$$
\begin{aligned}
\langle\tilde{\boldsymbol{\Omega}}, m \mid \boldsymbol{\Omega}, m\rangle= & \left(\text { terms containing }\left\langle\uparrow^{\prime} \mid \downarrow^{\prime}\right\rangle \text { and }\left\langle\downarrow^{\prime} \mid \uparrow^{\prime}\right\rangle\right) \\
& +\left(\cos \frac{\tilde{\theta}}{2} \cos \frac{\theta}{2}+\sin \frac{\tilde{\theta}}{2} \sin \frac{\theta}{2} e^{-i(\tilde{\phi}-\phi)}\right)^{s+m} \\
& \times\left(\sin \frac{\tilde{\theta}}{2} \sin \frac{\theta}{2} e^{i(\tilde{\phi}-\phi)}+\cos \frac{\tilde{\theta}}{2} \cos \frac{\theta}{2}\right)^{s-m} .
\end{aligned}
$$

First-order expansion in $\delta \theta$ and $\delta \phi$ leads to the overlap between two infinitesimally separated states, $\langle\tilde{\boldsymbol{\Omega}}, m \mid \boldsymbol{\Omega}, m\rangle \approx 1+i m(\cos \theta-1) \delta \phi . \quad$ After slicing the imaginary time interval $\beta$ into $N$ pieces of length $\epsilon=\beta / N, 14$ the transition amplitude $\left\langle\boldsymbol{\Omega}, m\left(\tau_{n+1}\right)|1-\epsilon \mathcal{H}| \boldsymbol{\Omega}, m\left(\tau_{n}\right)\right\rangle$ between two time steps $\tau_{n+1}$ and $\tau_{n}=n \epsilon$ can be approximated by $(1-$ $\epsilon \mathcal{H}[m \boldsymbol{\Omega}])\left\langle\boldsymbol{\Omega}, m\left(\tau_{n+1}\right) \mid \boldsymbol{\Omega}, m\left(\tau_{n}\right)\right\rangle$, where $\mathcal{H}[m \boldsymbol{\Omega}]$ is the diagonal element of the Hamiltonian. Collecting the various terms, we obtain

$$
\left\langle\tilde{\boldsymbol{\Omega}}, m\left|e^{-\beta \mathcal{H}}\right| \boldsymbol{\Omega}, m\right\rangle=\int \mathcal{D} \Omega e^{-i \Phi(m)-S_{\mathrm{dyn}}},
$$

where $S_{\text {dyn }}=\int_{0}^{\beta} d \tau \mathcal{H}[m \boldsymbol{\Omega}]$ is the dynamical action and $\Phi(m)=m \int_{-\pi / 2}^{\pi / 2} d \phi(1-\cos \theta)$ the Berry phase, with $\Phi(m) / m$ measuring the area of the spherical triangle between the tunneling path and the great circle through the north pole of $S^{2}$ (see Fig. 1).

Now we possess the tool to compute the path integral in Eq. (3) also for low-lying excited states $|m\rangle, m \lesssim s$. Since substitution of $s$ by $m$ in Eq. (2) does not change the symmetry of $E_{y, n}, S_{\text {dyn }}$ remains invariant under $C_{y, n}$ operations, whereas $\mathcal{R}_{y, \eta} \Phi(m) / m=\Phi(m) / m+4 \pi / n$. Thus, we can divide the $S^{2}$ surface area of integration in Eq. (7) into $n$ equally shaped subareas $\mathcal{A}_{n}$, so that we can factor out the following sum over Berry phase terms without the need of evaluating the dynamical part of the path integral (i.e., the following result is valid for all $m$ ),

$$
\left\langle-\frac{\pi}{2}\left|e^{-\beta \mathcal{H}_{y, n}}\right|+\frac{\pi}{2}\right\rangle \propto \sum_{k=1}^{n} e^{i(2 \pi / n)(2 k-1) m}=\frac{\sin (2 \pi m)}{\sin [(2 \pi / n) m]},
$$


which vanishes whenever $n$ is not a divisor of $2 m$. Thus, the tunnel splitting energy $E_{m,-m}$ between the states $|m\rangle$ and $|-m\rangle$ vanishes if $2 m / n \notin \mathbb{Z}$. However, if $2 m / n \in \mathbb{Z}$, the variable $m$ must be extended to real numbers, i.e., $m \rightarrow \mu \in \mathbb{R}$, in order to calculate the limit $\mu \rightarrow m$ of the ratio of the sine functions, which is plotted in Fig. 2 for a special case. In order to visualize the interference between the Berry phases in Eq. (8), we select one representative path of each subarea $\mathcal{A}_{n}$. Then the vanishing of the amplitude in Eq. (8) for the case $n=6$ (see Fig. 11) can be thought of as a destructive interference between six different paths. Note that there is no simple pairwise cancellation 11 Also, it is important to note that from the semiclassical point of view the total classical energy of the spin system $E$ must be conserved during the tunneling process. Therefore the semiclassical paths do not follow the local minima of $E$ with respect to $\theta$ alone, exceptfor the case of purely quadratic (i.e., $n=2$ ) anisotropies. 19.22

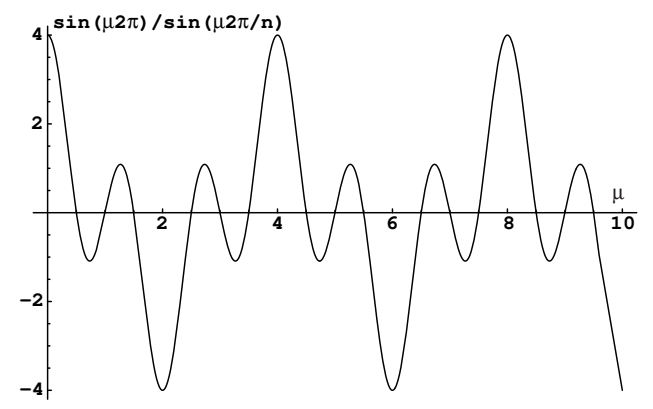

FIG. 2. Sum over Berry phase terms for $s=10$ and $n=4$.

From the above treatment we conclude that tunneling between two degenerate spin states $|m\rangle$ and $\left|m^{\prime}\right\rangle=|-m\rangle$ is topologically suppressed (i.e., the twofold degeneracy is not lifted) whenever $n$ is not a divisor of $2 m$. Since $n$ is even this excludes immediately tunneling for all halfodd integer spins $s$ (for all $m$ and $n$ ), in accordance with Kramers degeneracy. For the $s$ integer, however, tunneling can be either allowed or suppressed, depending on the ratio $2 m / n$. In the latter case, the twofold degeneracy of spin states is not lifted by the anisotropy, and we can view this result as a generalization of the Kramers theorem to integer spins. For the special case $n=2$, we immediately recover the spin-parity effect found before. 10

Now let us take a look at the tunnel splitting energy $E_{m m^{\prime}}$ by means of higher-order degenerate perturbation theory in terms of a standard spin operator formalism. Applying the resolvent technique we find

$E_{m m^{\prime}}=2\left|\frac{B_{n}^{\left(m-m^{\prime}\right) / n}}{\prod_{k=1}^{\left[\left(m-m^{\prime}\right) / n\right]-1} A\left[m^{2}-(m-k n)^{2}\right]}\right| \delta_{m-m^{\prime}, j n}$,

where $j \in \mathbb{Z}$. Note that $E_{m m^{\prime}}$ vanishes if $2 m / n \notin \mathbb{Z}$ for $m^{\prime}=-m$. This is a consequence of the fact that
$\mathcal{H}_{y, n}$ divides the Hilbert space $\mathbb{H}_{s}$ into a direct sum of invariant subspaces $h_{k}=\left\{\left|m^{\prime}\right\rangle \mid s-m^{\prime}=k \bmod n\right\}$, i.e., $\mathbb{H}_{s}=\bigoplus_{k=1}^{n} h_{k}$. Thus, the topological interference effects due to the Berry phase presented above correspond to these quantum-mechanical selection rules.

Next we take the external transverse magnetic field $H_{z}$ for the case $n=2$ into account. For this, we consider the following Hamiltonian $\left(A^{\prime}=A-2 B_{2}, B^{\prime}=4 B_{2}\right)$ :

$$
\mathcal{H}_{y, 2}=-A^{\prime} S_{y}^{2}+B^{\prime} S_{z}^{2}+h_{z} S_{z}+A^{\prime} m^{2} C,
$$

yielding the classical energy $E_{y, 2}(u)=$ $-A^{\prime} m^{2}\left[\left(1-u^{2}\right) \sin ^{2} \phi-C\right]+B^{\prime} m^{2} u^{2}+h_{z} m u$, where $h_{z}=g \mu_{B} H_{z}$ and $u=\cos \theta$. The copstant $C=1$ is determined by the boundary condition $\mathrm{g} u(\phi= \pm \pi / 2)=0$ for $h_{z}=0$. By completing the square in $E_{y, 2}(u)$ by the substitution $\tilde{u}=u+h_{z} / 2 m\left(A^{\prime} \sin ^{2} \phi+B^{\prime}\right)$, the Jacobian of which is unity, we end up with the anisotropy energy

$$
\begin{aligned}
E_{y, 2}(\tilde{u})= & -A^{\prime} m^{2}\left[\left(1-\tilde{u}^{2}\right) \sin ^{2} \phi-1\right]+B^{\prime} m^{2} \tilde{u}^{2} \\
& -\frac{1}{4} \frac{h_{z}^{2}}{A^{\prime} \sin ^{2} \phi+B^{\prime}} .
\end{aligned}
$$

$E_{y, 2}(\tilde{u})$ and the new integration limits for $\tilde{u}$ are invariant under $C_{y, 2}$ operations. Therefore, we can factor out two exponential terms with the following Berry phases:

$$
\begin{aligned}
\Phi_{ \pm}(m) & =m \int_{-\pi / 2}^{\pi / 2,-3 \pi / 2} d \phi\left(1+\frac{h_{z}}{2 m\left(A^{\prime} \sin ^{2} \phi+B^{\prime}\right)}\right) \\
& = \pm \pi m\left(1+\frac{h_{z}}{2 m \sqrt{B^{\prime}\left(A^{\prime}+B^{\prime}\right)}}\right)
\end{aligned}
$$

from which we directly obtain $\left\langle-\pi / 2\left|e^{-\beta \mathcal{H}_{y, 2}}\right|+\pi / 2\right\rangle \propto$ $\cos \left(\Phi_{+}\right)$and thus, Berry phase oscillations in $H_{z}$ with period $2 \sqrt{B^{\prime}\left(A^{\prime}+B^{\prime}\right)}=2 \sqrt{4 B_{2}\left(A+2 B_{2}\right)}$, and with phase shifts $\left|\Phi_{ \pm}(m)-\Phi_{ \pm}(m-1)\right|=\pi$, regardless between which states $|m\rangle$ and $|-m\rangle$ the tunneling takes place. Since $0 \leq\left|\Phi_{+}-\Phi_{-}\right| \leq 4 \pi m$, there are $2 m$ zeroes of the tunnel splitting $E_{m,-m} .18$ These findings agree with the exact diagonalization of Eq. (10) and have been observed in $\mathrm{Fe}_{8}$. . Note that these interference effects are due to the symmetry $C_{y, 2}$. Hence, when breaking this symmetry with a magnetic field that is tilted away from the $z$ axis (in the $x z$ plane) by the angle $\psi$ the oscillations become suppressed with increasing $\psi 19$ in agreement with Ref. 5. In contrast to previous work on $n=2$ (Ref. 12) we did not use approximations based on semiclassical paths (given by energy conservation_or on semiclassical Wentzel-Kramers-Brillouin methodse to find the zeroes of the tunnel splitting.

Next, we consider the case $n=4$,

$$
\mathcal{H}_{y, 4}=-A S_{y}^{2}+B_{4}\left(S_{+}^{4}+S_{-}^{4}\right)+h_{z} S_{z}+A m^{2} C,
$$

and its corresponding classical energy

$$
\begin{aligned}
E_{y, 4}= & -A m^{2}\left[\left(1-u^{2}\right) \sin ^{2} \phi-C\right]+2 B_{4} m^{4}\left[u^{4}-6 u^{2}\right. \\
& \left.\times\left(1-u^{2}\right) \cos ^{2} \phi+\left(1-u^{2}\right)^{2} \cos ^{4} \phi\right]+h_{z} m u .
\end{aligned}
$$


Again, the boundary conditions determine the constant $C=1$ for $h_{z}=0$. Unfortunately, it is no longer possible to complete the quartic polynomial in Eq. (14). The same problem persists for $n>4$. This means that in contrast to $n=2$, the $C_{y, 4}$ symmetry of the anisotropy energy $E_{y, 4}$ without transverse field $H_{z}$ cannot be restored anymore. However, there still remains the reflection symmetry between the conjugated paths $\gamma_{+}=(\theta, \phi)$ and $\gamma_{-}=(\theta,-\phi)$ that wind around the $z$ axis counterclockwise and clockwise, respectively. The topological part of the main contribution to the tunneling amplitude exhibits oscillations, as shown in Fig. 3. This part comes from the two semiclassical paths $u_{\mathrm{cl}, \pm}(\theta)$ that satisfy $E_{y, 4}=0$ and whose Berry phases, defined by

$$
\pm \Phi_{\mathrm{cl}}=m \int_{-\pi / 2}^{\pi / 2,-3 \pi / 2} d \phi\left(1-\operatorname{Re}\left\{u_{\mathrm{cl}, \pm}\right\}\right)
$$

interfere with each other. Thus, the level splitting induced by tunneling shows quantum oscillations as a function of $H_{z}$ due to this oscillating Berry phase 18 Again, for a magnetic field with $\psi \neq k \pi / 2, k \in \mathbb{Z}$, the reflection symmetry between conjugated paths is broken (see above), and therefore the more $\psi$ is detuned from $k \pi / 2$ the more the Berry phase oscillations are suppressed.19 Note that the level splitting contains $m$ zeroes, $m=$ 8, 9, 10, in the interval $-4 \mathrm{~T} \leq H_{z} \leq 4 \mathrm{~T}$ (see Fig. 3), and that the Berry phase oscillations are shifted by $\pi / 2$ when the parity of $m$ changes (see $m=8,9,10$ in Fig. 3), all in agreement with exact diagonalization 21 Thus, we have obtained a new spin-parity effect for $n=4$ in the case of integer spins $s$ which results from Berry phase oscillations. This effect should be observable for $\psi=k \pi / 2$ in molecular magnets such as $\mathrm{Mn}_{12}$. Since for all $n$ two conjugated paths (see above) can be found, Berry phase oscillations and their associated parity effect are maximal whenever $\psi=k 2 \pi / n 19$ This provides an experimental means to determine the order $n$ of transverse anisotropies.

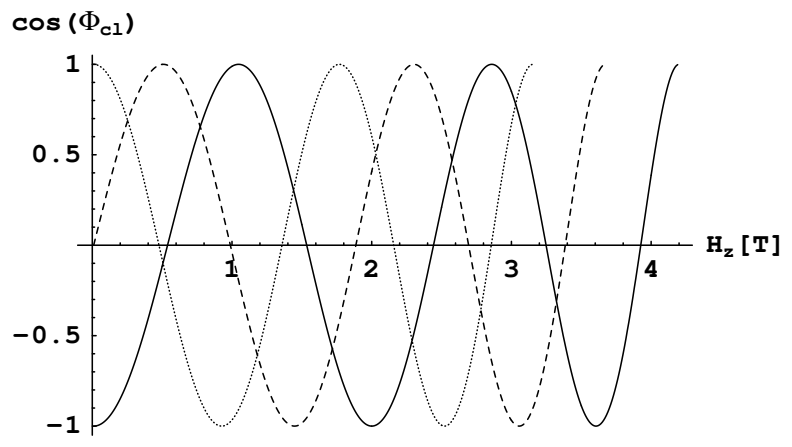

FIG. 3. Topological part of the tunneling amplitude for $n=4$ and $s=10$ as a function of $H_{z}$ [see Eq. (15)]. Solid line: $m=10$. Dashed line: $m=9$. Dotted line: $m=8$. The right boundary of $\cos \left(\Phi_{\mathrm{cl}}\right)$ is determined by $0 \leq 2\left|\Phi_{\mathrm{cl}}\right| \leq 4 \pi m$. The values $A=0.54 \mathrm{~K}$ and $B_{4}=8.5 \times 10^{-5} \mathrm{~K}$ approximate roughly the anisotropies in $\mathrm{Mn}_{12}$ (Ref. Ұ)
In conclusion, we have shown that the tunnel splitting vanishes due to interfering spin Berry phase and thus the level degeneracy is not lifted whenever $n$ is not a divisor of $2 m$. This generalization of the Kramers degeneracy to integer spins holds not only for the ground state but also for low-lying excited states $m$ and for transverse anisotropies of arbitrary order $n$; the presence of magnetic fields leads to oscillations of the tunnel splitting.

This work has been supported in part by the Swiss NSF and by Molnanomag HPRN-CT-1999-00012.

* Electronic address: Michael.Leuenberger@unibas.ch

† Electronic address: Daniel.Loss@unibas.ch

${ }^{1}$ C. Paulsen, J.-G. Park, B. Barbara, R. Sessoli, and A. Caneschi, J. Magn. Magn. Mater. 140-144, 379 (1995).

2 J. R. Friedman, M. P. Sarachik, J. Tejada, and R. Ziolo, Phys. Rev. Lett. 76, 3830 (1996).

${ }^{3}$ L. Thomas, F. Lionti, R. Ballou, D. Gatteschi, R. Sessoli, and B. Barbara, Nature (London) 383, 145 (1996).

${ }^{4}$ C. Sangregorio, T. Ohm, C. Paulsen, R. Sessoli, and D. Gatteschi, Phys. Rev. Lett. 78, 4645 (1997).

${ }^{5}$ W. Wernsdorfer and R. Sessoli, Science 284, 133 (1999).

${ }^{6}$ A. Fort, A. Rettori, J. Villain, D. Gatteschi, and R. Sessoli, Phys. Rev. Lett. 80, 612 (1998).

${ }^{7}$ M. N. Leuenberger and D. Loss, Europhys. Lett. 46, 692 (1999); Phys. Rev. B 61, 1286 (2000); Europhys. Lett. 52, 247 (2000).

${ }^{8}$ M. N. Leuenberger and D. Loss, Phys. Rev. B 61, 12200 (2000).

9 J. R. Klauder, Phys. Rev. D 19, 2349 (1979).

${ }^{10}$ D. Loss, D. P. DiVincenzo, and G. Grinstein, Phys. Rev. Lett. 69, 3232 (1992); H.-B. Braun and D. Loss, Phys. Rev. B 53, 3237 (1996).

11 J. von Delft and C. L. Henley, Phys. Rev. Lett. 69, 3236 (1992).

12 A. Garg, Europhys. Lett. 22, 205 (1993).

${ }^{13}$ V. A. Kalatsky and V. L. Pokrovsky, Europhys. Lett. 44, 539 (1998).

${ }^{14}$ E. Fradkin, Field Theories in Condensed Matter (AddisonWesley, Reading, MA, 1991).

15 A. Perelomov, Generalized Coherent States and Their Applications (Springer, New York, 1986).

${ }^{16}$ We note that the chosen parametrization makes the spin$1 / 2$ states single-valued everywhere on the sphere except at the south pole (i.e., the Dirac string is placed along the negative $z$ axis) (see Ref. 10).

${ }^{17}\langle\tilde{\boldsymbol{\Omega}}, m \mid \boldsymbol{\Omega}, m\rangle$ has a width of order $\sqrt{s}$ if $m$ is close to $s$.

${ }^{18}$ It is well known from instanton calculations (Ref. 10 ) that $E_{m,-m} \propto\left\langle-\pi / 2\left|e^{-\beta \mathcal{H}_{y, 2}}\right|+\pi / 2\right\rangle$ and thus $\propto\left|\cos \left(\Phi_{+}\right)\right|$.

${ }^{19}$ Changing the sign of $B_{n}$ is equivalent to applying $\mathcal{R}_{\alpha, \eta / 2}$ to $E_{\alpha, n}$. Thus for $B_{n}<0$ the Berry phase oscillations are maximal for $\psi=j \pi / n$, where $j$ is an odd integer.

${ }^{20}$ A. Garg, Phys. Rev. Lett. 83, 4385 (1999).

${ }^{21}$ I. Tupitsyn and B. Barbara, cond-mat/0002180 (unpublished); T. Pohjola and H. Schoeller, cond-mat/0005135 (unpublished). 